\title{
Melatonin Pretreatment Protects Against Status Epilepticus, Glutamate Transport, and Oxidative Stress Induced by Kainic Acid in Zebrafish
}

\section{Ana Caroline Salvador de Farias}

Universidade do Extremo Sul Catarinense

Karolyne de Pieri Pickler

Universidade do Extremo Sul Catarinense

Henrique Teza Bernardo

Universidade do Extremo Sul Catarinense

Samira Leila Baldin

Universidade do Extremo Sul Catarinense

\section{Eduardo Ronconi Dondossola}

Universidade do Extremo Sul Catarinense

\section{Eduardo Pacheco Rico ( $\nabla$ eduprico@gmail.com )}

Universidade do Sul Extremo Catarinense: Universidade do Extremo Sul Catarinense https://orcid.org/0000-0003-0019-328X

\section{Research Article}

Keywords: Epilepsy, Melatonin, Oxidative Stress, Glutamate, Zebrafish

Posted Date: June 17th, 2021

DOl: https://doi.org/10.21203/rs.3.rs-611477/v1

License: (c) (1) This work is licensed under a Creative Commons Attribution 4.0 International License. Read Full License

Version of Record: A version of this preprint was published at Molecular Neurobiology on October 19th, 2021. See the published version at https://doi.org/10.1007/s12035-021-02579-4. 


\section{Abstract}

Status epilepticus (SE) develops from abnormal electrical discharges, resulting in neuronal damage. Current treatments include antiepileptic drugs. However, the most common drugs used to treat seizures may sometimes be ineffective and have many side effects. Melatonin is an endogenous physiological hormone that is considered an alternative treatment for neurological disorders because of its free radical scavenging property. Thus, this study aimed to determine the effects of melatonin pretreatment on SE by inducing glutamatergic hyperstimulation in zebrafish. Seizures were induced in zebrafish using kainic acid (KA), a glutamate analog, and the seizure intensity was recorded for $60 \mathrm{~min}$. Melatonin treatment for 7 days showed a decrease in seizure intensity (28\%), latency to reach score 5 (14 min), and duration of SE (29\%). In addition, melatonin treatment attenuated glutamate transporter levels, which significantly decreased in the zebrafish brain after $12 \mathrm{~h}$ of KA-induced seizures. Melatonin treatment reduced the increase in oxidative stress by reactive oxygen species formation through thiobarbituric acid reactive substances and 2',7'-dichiorofluorescin, induced by KA-seizure. An imbalance of antioxidant enzyme activities such as superoxide dismutase and catalase was influenced by melatonin and KA-induced seizures. Our study indicates that melatonin promotes a neuroprotective response against the epileptic profile in zebrafish. These effects could be related to the modulation of glutamatergic neurotransmission, recovery of glutamate uptake, and oxidative stress parameters in the zebrafish brain.

\section{Highlights}

- Pre-treatment with melatonin reduces seizure intensity and Status epilepticus time in zebrafish.

- Pre-treatment with melatonin increases latency time for seizures, induced by kainic acid.

- TBA-RS and DCFH levels are attenuated in zebrafish pre-treated with melatonin.

- Glutamate uptake is reduced in control KA fish, reaching basal levels when pretreated with melatonin.

\section{Introduction}

Epilepsy is the second most common neurological disorder with the highest incidence worldwide, characterized by recurrent seizures and abnormal EEG recordings in the brain [1,2]. Epileptogenesis is the underlying process in which a normal brain becomes epileptic due to numerous factors such as trauma, genetics, infections, and metabolic abnormalities [3].

The glutamatergic system plays an important role in the initiation and propagation of Status epilepticus (SE) [4]. Seizures emerge from abnormal hyperexcitability and hypersynchronization of neurons, resulting in unprovoked excessive electrical discharges causing glutamatergic excitotoxicity, oxidative stress, and neuronal apoptosis [5]. Hyperexcitability leads to excessive glutamate release in the synaptic cleft, resulting in higher calcium influx that leads to mitochondrial dysfunction and a subsequent increase in oxidative stress [6]. 
Among multiple therapeutics targeted for the treatment of epilepsy, the use of antiepileptic drugs (AEDs) is the most common. These medications act on sodium ionic channels, GABAergic, and glutamatergic systems. The most common medications are sodium valproate, carbamazepine, and acetazolamide [7]. Current treatment involves the use of multiple drugs to attenuate seizures. However, if the dose is not appropriate [8], this approach can result in neurotoxic effects. Long-term use of AEDs can cause cognitive impairment and oxidative damage to the brain, such as modulation of antioxidant enzymes, stimulation of reactive oxygen species (ROS) production, and promotion of toxicity to essential macromolecules in the body [9]. It has been estimated that $30 \%$ of patients do not respond to treatment and furthermore, pharmacological resistance necessitates the search for alternative treatments to improve the quality of life in these patients $[10,11]$. The need to find alternative treatments and drugs is growing as treatmentresistant cases are increasing.

Melatonin (N-acetyl-5-methoxytryptamine) is an endogenous hormone with numerous properties including immunological, anti-inflammatory, reproductive, hormonal, and aging regulation [12]. Due to its amphiphilic properties, melatonin can easily cross the blood brain barrier, assisting in neuronal processes [13]. Melatonin attenuates cellular death resulting from oxidative damage by capturing free radicals and neutralizing, and turning some of the most dangerous ROS, such as $\mathrm{H}_{2} \mathrm{O}_{2}, \mathrm{O}_{2}^{-}, \mathrm{HO}^{-}, \mathrm{NO}, \mathrm{ONOO}$, and $\mathrm{HOCl}$, less reactive $[14,15]$. These properties of melatonin provide an interesting opportunity to investigate its effectiveness in neurological diseases.

Considering the translational neuroscience perspective, zebrafish is a suitable animal model for research on the mechanisms underlying seizures and epilepsy $[16,17]$. Behavioral and neurochemical screens in zebrafish offer an intriguing alternative preclinical approach to CNS drug discovery [18]. Furthermore, the glutamatergic system expresses excitatory amino acid transporters that facilitate maintenance of the neurotransmitter levels and uptake in the brain $[19,20]$. Studies have shown that an injection of kainic acid (KA) can generate seizure-like behavior and changes in glutamate transport, resulting in encephalic damage $[16,21]$.

Considering the cognitive, neuronal, psychological, and social impact of epilepsy and the percentage of patients refractory to treatment, the use of alternative neuroprotective molecules such as melatonin is relevant for treatment. Thus, we investigated the possible influence of melatonin pretreatment on KAinduced behavioral and neurochemical changes in adult zebrafish.

\section{Materials And Methods}

\section{Animals and housing conditions}

Adult wild-type Zebrafish (Danio rerio), of both sexes, obtained from the department of Biochemistry at Federal University of Rio Grande do Sul (UFRGS). Zebrafish were conditioned at the Translational Psychiatry laboratory of UNESC and maintained according to standard husbandry procedures. The water was kept at $26 \pm 2^{\circ} \mathrm{C}$ under $14 \mathrm{~h} / 10 \mathrm{~h}$ light dark cycle photoperiod and fed twice a day with artemia and 
commercial flake fish food. All procedures presented in this study were approved by the Ethics Committee of University of Southern Santa Catarina (UNESC) protocol number 030/2019-1.

\section{Melatonin exposure and seizure induction}

The molecule was administered directly into the aquariums half an hour before the lights went off in the zebrafish facility at a final concentration of $100 \mathrm{nM}$, according previous described [22]. Fish were exposed to melatonin in two different time periods, being: melatonin added for 3 nights, and 7 nights treatment in which seizures were induced the morning after each period (Fig. 1A). To ensure the same concentration of melatonin daily, the water in the tanks was completely exchanged every day. After melatonin treatment, fish were immersed in $160 \mu \mathrm{g} / \mathrm{mL}$ of tricaine and then inoculated $(10 \mu \mathrm{L})$ intraperitoneally (i.p.) with $\mathrm{KA}$ at $5 \mathrm{mg} / \mathrm{kg}$ [21]. The fish groups that were not induced seizures were similarly anaesthetized and injected with PBS. After seizure induction, fish were observed individually for a period of $60 \mathrm{~min}$. The seizures presents classic behaviors and abnormal brain activity, which can be observed through a numerical system of scores, varying from 1-7, being: 1 ) immobility and hyperventilation; 2 ) whirlpool-like swimming; 3) rapid movements from right to left; 4) abnormal and spasmodic muscular contractions, 5) rapid whole-body clonus-like convulsions; 6 ) sinking to the bottom of the tank and spasms for several minutes; 7) death [16]. Seizure intensity, latency, and $S E$ as duration of 5 and 6 score time was evaluated.

After the preliminary test verifying the effect of different times of melatonin exposure, we established 7 days of pretreatment for biochemical analyzes (Fig. 2A). For this, fish were divided into four groups according to each treatment: CTL + PBS, Mel + PBS, CTL + KA and Mel + KA. Control groups underwent the same procedures without the administration of any molecule into the water. Seizure- induced by KA generate neurochemical consequences after different periods, including glutamate transport and microglial markers [21]. In order to verify whether melatonin is able to influence the changes caused by KA-induced glutamatergic hyperstimulation, glutamate uptake and oxidative stress parameters were evaluated. Thus, the end of 12 hours post seizure induction the animals were anesthetized by immersing them in $160 \mathrm{mg} / \mathrm{ml}$ of tricaine $\left(4^{\circ} \mathrm{C}\right)$, suffered euthanasia and their brain contents were dissected for neurochemical analysis.

\section{Evaluation oxidative stress parameters}

Tissue preparation

Five zebrafish brains were pooled and used to prepare each homogenate fraction $(n=6)$. Tissues homogenized in $1 \mathrm{~mL}$ of $20 \mathrm{mM}$ sodium phosphate buffer, $\mathrm{pH} 7.4$, containing $140 \mathrm{mM} \mathrm{KCl}$. Homogenates were centrifuged at $750 \times \mathrm{g}$ for $10 \mathrm{~min}$ at $4^{\circ} \mathrm{C}$ to discard nuclei and cell debris [23]. The pellet was discarded, and the supernatant was collected and used for the evaluation of oxidative stress parameters.

Determination of oxidation of DCFH 
The production of reactive oxygen species (EROS) was measured from the oxidation of 2 ',7'dihydrodichlorofluorescein diacetate forming DCF, where the intensity of fluorescence is in accordance with the formation of EROS [24]. A calibration curve was performed with standard DCF $(0.25-10 \mu \mathrm{M})$ and the levels of reactive species were expressed as nmol DCF formed.mg of protein ${ }^{-1}$.

\section{Determination of TBA-RS levels}

The levels of substances reactive to thiobarbituric acid (TBA-RS) evaluate the lipid peroxidation produced by reactive oxygen species. This technique is based on the principle of species reactive to thiobarbituric acid forming a pink color in the samples [25]. A calibration curve was established using 1,1,3,3tetramethoxypropane and each curve point was subjected to the same treatment as supernatants. TBARS values were measured and expressed as nmol of TBA-RS.mg protein ${ }^{-1}$.

Antioxidant enzyme activities determination

Determination of SOD and CAT activities

Superoxide Dismutase (SOD) was carried out using methods already established, based on the oxidation of adrenaline by the enzyme $\mathrm{SOD}$, disabling the dismutation of the radical $\mathrm{O}_{2}^{-}$in $\mathrm{H}_{2} \mathrm{O}_{2}$ [26]. The reaction medium consisted of $50 \mathrm{mM}$ glycine buffer, $\mathrm{pH}$ 10.2, $0.1 \mathrm{mM}$ catalase and $1 \mathrm{mM}$ epinephrine. Absorbance was measured at $480 \mathrm{~nm}$. SOD specific activity is expressed as nmol. $\mathrm{min}^{-1} \cdot \mathrm{mg} \mathrm{protein}^{-1}$. Catalase (CAT) activity assay was performed through the measuring of absorbance decrease at $240 \mathrm{~nm}$ in a reaction medium containing $20 \mathrm{mM} \mathrm{H}_{2} \mathrm{O}_{2}, 0.1 \%$ Triton X-100, $10 \mathrm{mM}$ potassium phosphate buffer, $\mathrm{pH} 7.0$, and the supernatants containing $0.1-0.3 \mathrm{mg}$ protein. $\mathrm{mL}^{-1}$ [27]. The specific activity was represented as nmol. $\mathrm{min}^{-1} . \mathrm{mg}$ protein ${ }^{-1}$. The set of two enzymatic activities were expressed using the SOD/CAT ratio.

Determination of GR and GPx activities.

Glutathione reductase (GR) is responsible for catalyzing the GSSG reduction reaction in NADPHdependent GSH [28]. The enzyme activity was assessed in a solution containing $50 \mathrm{mM}$ potassium phosphate buffer, $\mathrm{pH} 7.0$, containing $1 \mathrm{mM}$ EDTA, $0.2 \mathrm{mM} \mathrm{NADPH}$ and the supernatant containing 0.3$0.5 \mathrm{mg}$ protein $\cdot \mathrm{ml}^{-1}$. The reaction was initiated by the addition of $1 \mathrm{mM}$ oxidized glutathione and a change in absorbance was measured at $340 \mathrm{~nm}$. GR activity was expressed as nmol NADPH oxidized. $\mathrm{min}^{-1}$.mg protein ${ }^{-1}$. The enzymes Glutathione peroxidases (GPx), are responsible for the consumption of NADPH to generate reduced glutathione (GSH) from oxidized glutathione (GSSG), acting on the metabolism of $\mathrm{H}_{2} \mathrm{O}_{2}$ [29]. This method was performed using Tert-butylhydroperoxide as a substrate. The enzyme activity was determined by monitoring the NADPH disappearance at $340 \mathrm{~nm}$ in 50 $\mathrm{mM}$ potassium phosphate buffer, $\mathrm{pH} 7.0$, containing $1 \mathrm{mM}$ EDTA, $1 \mathrm{mM}$ glutathione, $0.2 \mathrm{U}^{\mathrm{ml}} \mathrm{m}^{-1}$ glutathione reductase, $1 \mathrm{mM}$ azide, $0.2 \mathrm{mM}$ tert-butyl-hydroperoxide, $0.2 \mathrm{mM} \mathrm{NADPH}$ and the supernatant 
containing 0.2-0.3 mg protein.ml-1. GPx activity was expressed as nmol NADPH oxidized.min ${ }^{-1} . \mathrm{mg}^{-1}$ $\operatorname{protein}^{-1}$.

\section{Glutamate uptake assay}

Glutamate uptake assay was performed as previously described [20, 29]. The brains were set in microplates of 24 wells and total glutamate uptake was measured with the addition of $0.33 \mu \mathrm{Ci} \mathrm{mL} \mathrm{L}^{-1} \mathrm{~L}$ $\left[{ }^{3} \mathrm{H}\right]$ glutamate (PerkinElmer, Madrid, Spain) to the incubation medium (HBSS-HEPES buffer) pH 7.2 at $37^{\circ} \mathrm{C}$ for 7 minutes. After this period, the uptake was stopped with two subsequent washes with $1 \mathrm{~mL}$ icecold HBSS-HEPES buffer). Na+-independent glutamate uptake was measured using the same conditions as described above, except that N-methyl-D-glucamine was used instead of sodium. Na+-dependent glutamate uptake was measured as the difference of incorporated radioactivity between the total glutamate uptake and the $\mathrm{Na}$-independent glutamate uptake. Radioactivity was measured by liquid scintillation and uptake was expressed as nmol $\left[{ }^{3} \mathrm{H}\right]-\mathrm{Glu}_{\mathrm{min}}{ }^{-1} . \mathrm{mg}$ of protein $\left.{ }^{-1}\right)$.

\section{Protein determination}

Total protein quantification in the samples was performed by Lowry et al. (1951)[31] method using bovine serum albumin as standard.

\section{Statistical Analysis}

Statistical analyzes were performed using the statistical program SPSS Statistics (Armonk, New York, USA). The construction of all the graphs present in this study was performed using the Graph Pad Prism software version 8.4. The results were found as mean \pm standard error of the mean. The results were evaluated by one- or two-way analysis of variance (ANOVA) in which the degree of significance was evaluated, where $p<0.05$. As a result, where there was a difference, the Tukey test was performed as a post hoc test when necessary.

\section{Results}

The seizure-related behavioral phenotypes were assessed and all fish injected with KA presented with at least one rapid whole-body clonus-like convulsion, classified as a score of 5. To establish the standard conditions for melatonin exposure, zebrafish were initially pretreated for 3 and 7 days. Figure 2 shows seizure intensity over $60 \mathrm{~min}$. The data profile shows that the pretreated groups had decreased seizure intensities compared to KA-exposed fish (Fig. 2A). Melatonin pretreatment for 7 days significantly decreased the total seizure score as estimated by the area under the curve $\left(F_{(4,109)}=13.78, p<0.05\right)$ (Fig. $2 B$ ). Figure $2 \mathrm{C}$ shows the representative heat maps of individual scores of zebrafish for 60 min after $\mathrm{KA}$ injection. The distribution of the individual score values is indicative of changes in the latency time to 5 and the SE duration. The control KA group presented a latency time of $8.5 \pm 1.18 \mathrm{~min}$ to reach a score of 5 (Fig. 3A). Pretreatment of melatonin for 7 days significantly increased the latency to score 5 to $22 \pm 3$ min in comparison to the CTL + KA group $\left(F_{(2,68)}=6,061, p<0.01\right)$. Three days of exposure did not 
significantly alter this parameter. In addition to reducing the time to reach stage 5 , melatonin also affected the duration, $\mathrm{F}_{(2,89)}=3,102, p<0.01$. CTL + KA group exhibited $41 \pm 3.72$ min of SE duration, spending $68 \%$ of the time in seizure like behavior, transitioning between scores 5 and 6 (Fig. 3B). SE duration showed a significant decrease for Mel 3 days + KA and Mel 7 days + KA with 28.6 4.29 (31\%, $p$ $<0.05)$ and $29.6 \pm 4.37(29 \%, p<0.05)$ min, respectively.

Considering that zebrafish pretreated with melatonin for 7 days showed a significant decrease in SE duration with a significant increase in latency to reach score 5 , this time period was adopted to investigate glutamate neurotransmission and oxidative stress. To investigate whether melatonin modulated glutamate transport, we assessed glutamate uptake in the brain tissue of animals pretreated with melatonin for 7 days (Fig. 4). One-way ANOVA revealed significant differences between the tested groups. The CTL + PBS and Mel + PBS groups showed similar values for glutamate uptake, indicating that melatonin in isolation did not influence glutamate transport $\left(\mathrm{F}_{(3,22)}=3,529, p<0.05\right)$. CTL $+\mathrm{KA}$ showed a significant decrease $(37.7 \%)$ compared to CTL + PBS $(p<0.05)$. Interestingly, the Mel + KA group showed recovery in glutamate uptake, displaying a significant difference in comparison with CTL + KA $(p<0.05)$, similar to CTL + PBS.

Reduced glutamate uptake is associated with excitotoxicity and oxidative damage. Furthermore, a return to basal levels of glutamate uptake in fish pretreated with melatonin could show up as antioxidant neuroprotection. In order to evaluate oxidative damage, we assessed the free radical formation through TBA-RS and DCFH oxidation (Fig. 5). Regarding DCFH oxidation, two-way ANOVA using treatment and KA as variable factors showed significant effects of treatment $\left(F_{(4,54)}=18.82, p<0.001\right)$. No alterations were observed in DCFH levels in CTL + PBS, compared to Mel + PBS pretreated zebrafish, indicating that melatonin in isolation does not modulate the biomarkers of oxidative damage (Fig. 5A). Additionally, CTL + KA significantly increased DCFH oxidation by $71.1 \%$ compared to CTL +KA $(p<0.05)$, demonstrating that KA administration causes oxidative damage to the zebrafish brain. Furthermore, Mel $+\mathrm{KA}$ showed a significant decrease of $30 \%$ in DCFH oxidation compared to CTL $+K A(p<0.05)$. Lipid peroxidation was evaluated by measuring the levels of TBA-RS after melatonin and KA-induced seizures, which showed a significant effect $\left(F_{(4,54)}=92.14, p<0.0001\right)$ (Fig. 5B). TBA-RS levels showed a significant increase of $62.7 \%$ in the CTL + KA group, compared to the CTL + PBS group $(p<0.05)$. These results demonstrate that seizures themselves generate oxidative damage and increase free radical formation in the brain. $A$ decrease in TBA-RS levels (17.75\%) was observed in the Mel + KA group compared to that in the control $\mathrm{KA}$ group. To evaluate the underlying antioxidant properties of melatonin, we assayed antioxidant enzymes such as, SOD, CAT, GR, and GPx (Fig. 6). SOD activity was significantly lower in the Mel + PBS group than in the CTL + PBS group (36.5\%; $p<0.05)$ (Fig. 6A). Furthermore, enzyme activity also decreased significantly for Mel + KA compared to CTL + PBS $(26.5 \% ; p<0.05)$. Melatonin pretreatment and KA-induced seizures altered the CAT activity $\left(\mathrm{F}_{(3,20)}=4,552 ; p<0.05\right)(\mathrm{Fig} .6 \mathrm{~B})$. A decrease in CAT activity was observed for Mel + PBS (43.1\%; $p<0.01)$ and Mel + KA $(38.46 \% ; p<0.05)$, respectively. We evaluated the activities of CAT and SOD in the decomposition of superoxide radical anions and dismutation of hydrogen peroxide, respectively, using the SOD/CAT ratio. Pretreatment with Mel reversed 
the KA-induced imbalance in CAT/SOD to the basal level (Fig. 6C). No alterations were observed in GR and GPx activity in any of the groups tested (Fig. 6D-E).

\section{Discussion}

This is the first study to investigate the neuroprotective effects of the pretreatment with melatonin against $S E$ induced by KA in zebrafish. Melatonin is an important natural hormone responsible for the regulation of essential physiological processes in the organism. It is able to easily cross the blood-brain barrier and act as a potent antioxidant, with clinical uses in numerous diseases, and most recently in epilepsy studies $[13,32]$.

In this study, we first addressed the intensity of seizure behavior. Classic seizure movements were recorded and characterized for a period of $60 \mathrm{~min}$ through well-established assays in literature [16]. Our findings show that control KA fish spent an average of $41 \mathrm{~min}$ in $S E$, with extremely intense seizures, and a short latency time. These findings implicate that injection of KA in the zebrafish brain results in a hyperexcitability model, presenting cellular changes, and classic seizure-like behavior, which is consistent with previous studies in the zebrafish brain $[16,21]$.

Zebrafish pretreated with melatonin exhibited a decrease in seizure intensity and $S E$ duration, and an increase in latency to reach score 5 . These findings corroborate with previous studies, in which melatonin elicited neuroprotective responses through retardation of seizures, cognitive function, and electroencephalographic brain activity in a mammalian kainate model of temporal lobe epilepsy [33, 34]. To establish the conditions for melatonin pretreatment, we investigated different periods of exposure. We found that 7 days of melatonin exposure showed a significantly higher protective response in the seizure score profile, compared to 3 days of pretreatment. Furthermore, the significant increase in latency to achieve tonic-clonic seizure, duration of scores 5 and 6 , and individual heat maps of scores confirmed the reduction of seizure profile. Establishment of these suitable conditions including period and concentration can be useful for understanding and exploring the neurophysiological and neurochemical properties of melatonin from the translational perspective.

The glutamatergic system plays a key role in the imbalance of excitatory and inhibitory synapses during $S E$. As an endogenous neuroendocrine neuromodulator, melatonin exerts a positive control on glutamate excitotoxicity that leads to receptor desensitization by decreasing glutamate uptake, resulting in excessive $\mathrm{Ca}^{2+}$ influx into neurons, causing oxidative stress and cellular damage which are the underlying mechanisms of neuronal impairment [35]. We found a decrease in $\mathrm{Na}^{+}$-dependent glutamate transporters $12 \mathrm{~h}$ after KA-induced zebrafish seizures. These data are in accordance with this time window, in which $12 \mathrm{~h}$ post-seizure there is disruption of glutamate uptake and alteration of astrocytic markers such as GFAP and S100 in the zebrafish brain [21]. In this context, we investigated whether melatonin treatment could counteract glutamatergic excitotoxicity in zebrafish in accordance with the reduction of seizure profiles and in the amelioration of episodes of seizures in animal models [36] and human [37]. Melatonin pretreatment alone did not modify the glutamate uptake. In contrast, zebrafish subjected to KA-induced 
seizures and previously treated with melatonin recovered $\mathrm{Na}$-dependent glutamate uptake similar to that in the untreated PBS group. These results suggest that melatonin can influence the disruption of this parameter, which is responsible for controlling glutamate homeostasis in the brain tissue. These pieces of evidence can be associated with the control of oxidative balance and the possible antioxidant properties of melatonin in zebrafish due to its ease in crossing the blood-brain barrier, resulting in facilitated access to neurons.

To evaluate the underlying processes of $S E$ behavioral profile and glutamate uptake, we assessed the oxidative stress parameters. Here, KA-induced seizures increased TBA-RS and DCFH in the zebrafish brain, resulting in free radical formation and higher levels of biomarkers of oxidative damage, similar to the findings in rat epilepsy models [38]. Furthermore, these phenomena corroborate previous studies, which suggest that epilepsy patients show higher levels of oxidative stress biomarkers, elevated ROS formation, reduced antioxidant enzyme activity, and vulnerability to oxidative brain damage [39, 40]. Our results show that control KA-injected zebrafish have increased lipoperoxidation and ROS formation and release in the brain, suggesting that oxidative stress could be related to glutamatergic disruption. Additionally, 7 days of melatonin pretreatment followed by seizure induction reduced the DCFH and TBARS levels, implying that melatonin is able to attenuate ROS formation in the zebrafish brain. Indeed, melatonin is a highly potent natural antioxidant with free radical scavenging properties, due to its electron-rich chemical structure, which makes these molecules less reactive [41]. Therefore, melatonin has a promising neuroprotective response against oxidation in the cerebral tissue of zebrafish, which can be associated with the neutralization of increased ROS formation in this SE glutamatergic model.

To further understand the mechanisms underlying the antioxidant defense of melatonin, we assessed the enzyme activities of SOD, CAT, GR, and GPx. Our findings showed an inhibition of SOD and CAT activities when melatonin was tested without SE induction and KA-induced seizures, compared to the control. A reduction in antioxidant enzyme activity or an imbalance in the redox system leads to oxidative stress $[42,43]$. SOD and CAT have a synergistic effect under physiological conditions, which play an important role in the regulation of superoxide anions $\left(\mathrm{O}_{2}\right)$ and $\mathrm{H}_{2} \mathrm{O}_{2}$. We assessed the SOD/CAT ratios and observed that melatonin treatment reversed the KA-induced imbalance in antioxidant enzyme activities. Based on this, the results found in this study could indicate that melatonin individually reduces enzyme activity, favoring an oxidative stress event. However, DCFH and TBA-RS results showed that melatonin reduced ROS formation. Melatonin and its metabolites are well-known antioxidant molecules that act as ROS scavengers. The mitigation of KA-induced seizure intensity, glutamate uptake, and oxidative stress by melatonin pretreatment suggests that its effects may influence circulation of ROS in the brain, reducing formation free radical formation, thereby reducing antioxidant enzyme activity in the zebrafish brain.

Lastly, to our knowledge, this is the first study to evaluate pretreatment with melatonin for SE induced by KA by modulating glutamatergic neurotransmission, recovering glutamate uptake, and oxidative stress in zebrafish. Although most epileptic patients develop pharmacologically resistant AEDs, alternative models could contribute to the search for new treatment strategies. The findings presented in this study can be 
used to understand the neuroprotective processes/properties of melatonin in the epileptic brain and contribute to finding a possible treatment from a translational perspective.

\section{Abbreviations}

2',7'-dihydrodichlorofluorescein diacetate (DCF); catalase (CAT); central nervous system (CNS); glutathione peroxidase (GPx); glutathione reductase (GR); superoxide dismutase (SOD); thiobarbituric acid-reactive species (TBA-RS).

\section{Declarations}

\section{Author contribution}

Ana Caroline Salvador de Farias: Investigation, Validation, Data curation, Writing - original draft. Karolyne de Pieri Pickler: Investigation, Validation, Data curation, Writing - original draft. Henrique Teza Bernardo: Conceptualization, Concept and design. Samira Leila Baldin: Formal analysis Investigation Writing review \& editing. Eduardo Ronconi Dondossola: Data analysis and interpretation. Eduardo Pacheco Rico: Funding acquisition, Project administration, Supervision, Writing - review \& editing, Resources. The paper was read, revised, and approved by all the authors.

\section{Data Availability}

The authors confirm that the data supporting the findings of this study are available within the article. The raw data are available from Eduardo Pacheco Rico upon reasonable request. The study protocol was approved by the Ethics Committee of University of Southern Santa Catarina (UNESC), Criciúma, Brazil, number 030/2019-1.

\section{Consent to Participate}

All participants gave written informed consent and signed by them. All consents are available upon request.

\section{Consent for Publication}

Not applicable.

\section{Conflict of Interest}

There are no competing interests.

\section{Acknowledgements}

This work was supported by Conselho Nacional de Desenvolvimento Científico e Tecnológico (CNPq) research Grant (Universal 429302/2018-5), Fundação de Amparo à Pesquisa do Estado de Santa 
Catarina (FAPESC - Universal 12/2020), and Coordenação de Aperfeiçoamento de Pessoal de Nível Superior (CAPES AUXPE PROEX N. 23038.020053/2018-52).

\section{References}

1. Epilepsy (2020). World Health Organization. https://www.who.int/news-room/factsheets/detail/epilepsy. Accessed 15 May 2020.

2. Ngugi, AK, Bottomley C, Kleinschmidt I. Sander JW, Newton CR (2010). Estimation of the burden of active and life-time epilepsy: A meta-analytic approach. Epilepsia 51: 883-890. https://doi.org/10.1111/j.1528-1167.2009.02481.x.

3. Goldberg, EM, Coulter DA (2013). Mechanisms of epileptogenesis: a convergence on neural circuit dysfunction. Nat Rev Neurosci. 14: 337-349. doi: 10.1038/nrn3482.

4. Zhou Y, Danbolt NC (2014). Glutamate as a neurotransmitter in the healthy brain. J Neur Transm. 121: 799-817. doi: 10.1007/s00702-014-1180-8.

5. Danbolt NC (2001). Glutamate uptake. Prog Neurobiol. 65: 1-105. doi: 10.1016/s03010082(00)00067-8. PMID: 11369436.

6. Vishnoi S, Raisuddin S, Parvez S (2016). Glutamate Excitotoxicity and Oxidative Stress in Epilepsy: Modulatory Role of Melatonin. J Environ Pathol Toxicol Oncol. 35: 365-374. doi: 10.1615/JEnvironPatholToxicolOncol.2016016399.

7. Rogawski MA, Löscher W (2004). The neurobiology of antiepileptic drugs. Nat Rev Neurosci. 5: 553564. doi: $10.1038 / \mathrm{nrn} 1430$.

8. Kwan P, Brodie MJ (2006). Combination Therapy in Epilepsy. Drugs. 66: 1817-1829. doi: 10.2165/00003495-200666140-00004.

9. Hamed SA, Abdellah MM (2004). Trace elements and electrolytes homeostasis and their relation to antioxidant enzyme activity in brain hyperexcitability of epileptic patients. J Pharmacol Sci. 96: 349359. doi: 10.1254/jphs.crj04004x.

10. Kwan P, Schachter SC, Brodie MJ (2011). Drug-resistant epilepsy. N Engl J Med. 365: 919-926. doi: 10.1056/NEJMra1004418. PMID: 21899452.

11. Sheng J, Liu S, Qin H, Li B, Zhang X (2018) Drug-Resistant Epilepsy and Surgery. Curr Neuropharmacol. 16 :17-28. doi: 10.2174/1570159X15666170504123316.

12. Zhdanova IV, Tucci V (2003). Rhythms, and Sleep. Curr Treat Options Neurol. 5: 225-229. doi: 10.1007/s11940-003-0013-0.

13. Alghamdi BS (2018). The neuroprotective role of melatonin in neurological disorders. Journ of Neurosci Res. 96:1136-1149. doi: 10.1002/jnr.24220.

14. Tan DX, Manchester LC, Esteban-Zubero E, Zhou Z, Reiter RJ (2015). Melatonin as a potent and inducible endogenous antioxidant: Synthesis and metabolism. Molecules. 20:18886-18906. doi: 10.3390/molecules201018886. 
15. Tarocco A, Caroccia N, Morciano G, Wiecksowki MR, Ancora G, Garani G, Pinton P (2019). Melatonin as a master regulator of cell death and inflammation: molecular mechanisms and clinical implications for newborn care. Cell Death Dis. 10:317. doi: 10.1038/s41419-019-1556-7.

16. Alfaro JM, Ripoll-Gómez, J, Burgos JS (2011). Kainate administered to adult zebrafish causes seizures similar to those in rodent models. Europ J of Neurosci. 33:1252-1255. doi: 10.1111/j.14609568.2011.07622.x.

17. Fontana BD, Ziani PR, Canzian J, Mezzomo NJ, Muller TE, dos Santos MM, Loro VL, Barbosa NV, Mello CF, Rosemberg DB (2019). Taurine Protects from Pentylenetetrazole-Induced Behavioral and Neurochemical Changes in Zebrafish. Mol Neurobiol. 56:583-594. doi: 10.1007/s12035-018-1107-8.

18. Wyatt C, Bartoszek EM, Yaksi E (2015). Methods for studying the zebrafish brain: Past, present and future. Eur J Neurosci. 42: 1746-1763. doi: 10.1111/ejn.12932.

19. Guo, S (2009). Using zebrafish to assess the impact of drugs on neural development and function. Expert Opin Drug Discov. 4: 715-726. doi: 10.1517/17460440902988464.

20. Rico EP, de Oliveira DL, Rosemberg DB, Mussulini BH, Bonan CD, Dias RD, Wofchuck S, Souza DO, Bogo MR (2010). Expression and functional analysis of Na+-dependent glutamate transporters from zebrafish brain. Brain Res Bull. 81: 517-523. https://doi.org/10.1016/j.brainresbull.2009.11.011

21. Mussulini BHM, Vizuete AFK, Braga M, Moro L, Baggio S, Santos E, Lazzarotto G, Zenki KC, Pettenuzzo L, Rocha JBT, de Oliveira DL, Calcagnotto ME, Zuanazzi JAS, Burgos JS, Rico EP (2018). Forebrain glutamate uptake and behavioral parameters are altered in adult zebrafish after the induction of Status Epilepticus by kainic acid. NeuroToxicol. 67: 305-312. https://doi.org/10.1016/j.neuro.2018.04.007

22. Pinheiro-Da-Silva J, Tran S, Luchiari AC (2018). Sleep deprivation impairs cognitive performance in zebrafish: A matter of fact? Behav Processes. 157:656-663. doi: 10.1016/j.beproc.2018.04.004.

23. Agostini JF, Dal Toé HCZ, Vieira KM, Baldin SM, Costa NLF, Cruz CU, Longo L, Machado MM, da Silveira TR, Schuck PF, Rico EP (2017). Cholinergic System and Oxidative Stress Changes in the Brain of a Zebrafish Model Chronically Exposed to Ethanol. Neurotox Res 33:749-758. doi: 10.1007/s12640-017-9816-8.

24. Lebel CP, Ischiropoulos H, Bondy SC (1992). Evaluation of the Probe 2',7'-Dichlorofluorescin as an Indicator of Reactive Oxygen Species Formation and Oxidative Stress. Chem Res Toxicol. 5:227231. doi: 10.1021/tx00026a012.

25. Esterbauer H, Cheeseman, KH (1990). Determination of aldehydic lipid peroxidation products: Malonaldehyde and 4-hydroxynonenal. Method in Enzymol. 186:407-421. doi: 10.1016/00766879(90)86134-h.

26. Bannister JV, Calabrese L (1987). Assays for superoxide dismutase. Methods biochem anal 32:279312. doi: 10.1002/9780470110539.ch5.

27. Aebi H (1984). Catalase in Vitro. Methods Enzymol. doi: 10.1016/s0076-6879(84)05016-3.

28. Carlberg I, Mannervik B (1985) Glutathione reductase. Methods Enzymol. 113: 484-490. doi: 10.1016/s0076-6879(85)13062-4. PMID: 3003504. 
29. Wendel A (1981). Glutathione Peroxidase. Methods Enzymol. 77:325-333. doi: 10.1016/s00766879(81)77046-0.

30. Baggio S, Mussulini BH, de Oliveira DL, Zenki KC, Santos da Silva E, Rico EP (2017) Embryonic alcohol exposure promotes long-term effects on cerebral glutamate transport of adult zebrafish. Neurosci Lett. 636: 265-269. doi: 10.1016/j.neulet.2016.11.016.

31. Lowry OH, Rosebrough NJ, Farr AL, Randall RJ (1951) Protein measurement with the Folin phenol reagent. J Biol Chem. 193: 265-725.

32. Khan S, Khurana M, Vyas P, Vohora D (2021) The role of melatonin and its analogues in epilepsy. Rev Neurosci. 32: 49-67. https://doi.org/10.1515/revneuro-2019-0088.

33. Tchekalarova J, Petkova Z, Pechlivanova D, Moyanova S, Kortenska L, Mitreva R, Lozanov V, Atanasova D, Lazarov N, Stoynev A (2013). Prophylactic treatment with melatonin after status epilepticus: Effects on epileptogenesis, neuronal damage, and behavioral changes in a kainate model of temporal lobe epilepsy. Epilepsy and Behav. 27:174-187. doi:

10.1016/j.yebeh.2013.01.009.

34. Zhdanova IV, Yu L, Lopez-Patino L, Shang E, Kishi S, Guelin E (2008). Aging of the circadian system in zebrafish and the effects of melatonin on sleep and cognitive performance. Brain Res Bull. 75: 433-441. doi: 10.1016/j.brainresbull.2007.10.053.

35. Mahmood D, Muhammad BY, Alghani M, Anwar J, el-Lebban N, Haider M (2016). Advancing role of melatonin in the treatment of neuropsychiatric disorders. Egyptian J Bas Appl Sci. 3:203-218.

36. Costa-Lotufo LV, Fonteles MMF, Lima ISP, de Oliveira AA, Nascimento VS, Bruin VMS, Viana GSB (2002). Attenuating effects of melatonin on pilocarpine-induced seizures in rats. Comp Biochem Physiol C Toxicol Pharmacol. 131:521-529. doi: 10.1016/s1532-0456(02)00037-6.

37. Sanchez-Barcelo EJ, Rueda N, Mediavilla MD, Martinez-Cue C, Reiter RJ (2017). Clinical Uses of Melatonin in Neurological Diseases and Mental and Behavioural Disorders. Curr Med Chem. 35: 3851-3878. doi:10.2174/0929867324666170718105557.

38. Gluck MR, Jayatilleke E, Shaw S, Rowan AJ, Haroutunian V (2000). CNS oxidative stress associated with the kainic acid rodent model of experimental epilepsy. Epilepsy Res. 39:63-71. doi: 10.1016/s0920-1211(99)00111-4.

39. Turkdogan D, Toplan S, Karakoc Y. Lipid peroxidation and antioxidative enzyme activities in childhood epilepsy. J Child Neurol. 2002 Sep;17(9):673-6. doi: 10.1177/088307380201700904. PMID: 12503643.

40. Ersan S, Cigdem B, Bakir D, Dogan HO (2020) Determination of levels of oxidative stress and nitrosative stress in patients with epilepsy. Epilepsy Res. 164: 106352. doi: 10.1016/j.eplepsyres.2020.106352.

41. Mareš J, Stopka P, Nohejlová K, Rokyta R (2013) Oxidative stress induced by epileptic seizure and its attenuation by melatonin. Physiol Res. 62: 67-74. doi: 10.33549/physiolres.932576. PMID: 24329705. 
42. Khan TA, Hassan I, Ahmad A, Perveen A, Aman S, Quddusi S, Alhazza IM, Ashraf GM, Aliev G. (2016) Recent Updates on the Dynamic Association Between Oxidative Stress and Neurodegenerative Disorders. CNS Neurol Disord Drug Targets. 15: 310-320. doi: 10.2174/1871527315666160202124518. PMID: 26831262.

43. Uttara B, Singh AV, Zamboni P, Mahajan RT (2009) Oxidative stress and neurodegenerative diseases: a review of upstream and downstream antioxidant therapeutic options. Curr Neuropharmacol. 7: 6574. doi: 10.2174/157015909787602823. PMID: 19721819; PMCID: PMC2724665.

\section{Figures}

Fig.1A-Evaluation of different time exposure of melatonin in KA induced-seizure in zebrafish
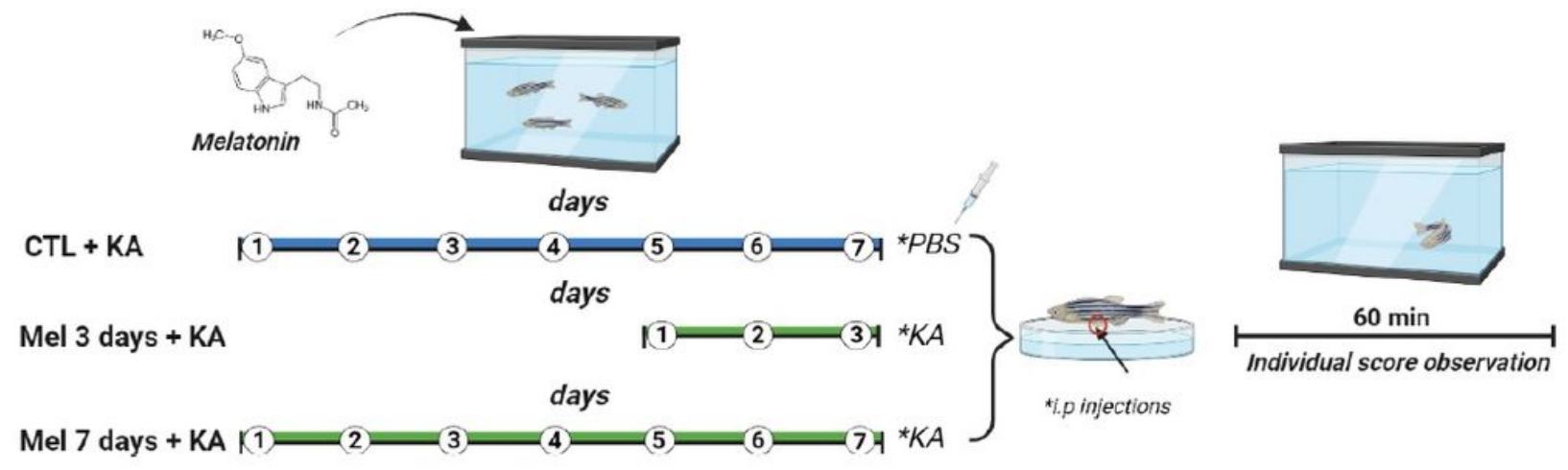

Fig.1B-Schematic overview of 7 days melatonin pretreatment in KA-induced seizures in zebrafish

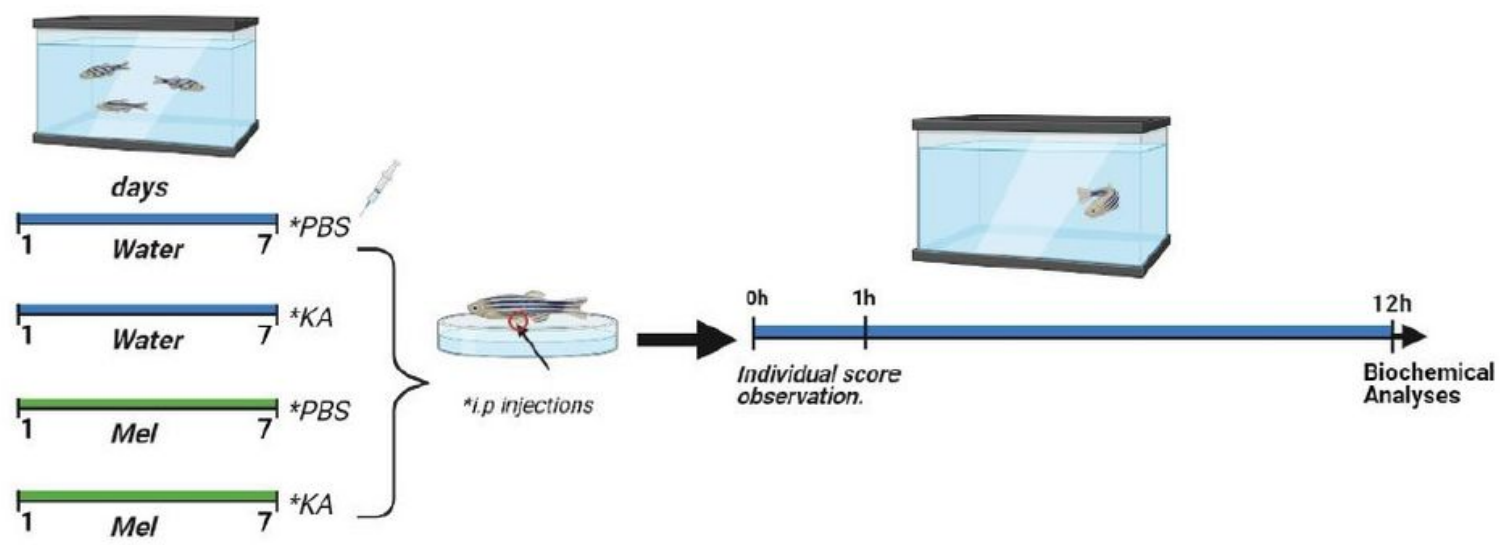

\section{Figure 1}

Schematic overview of the timeline for melatonin pretreatment in KA-induced seizures in zebrafish: Figure $1 \mathrm{~A}$ depicts different periods of melatonin exposure, followed by an analysis of seizure intensity for 60 min. Figure 1B shows the timeline for different groups exposed for 7 days with or without melatonin, followed by KA or PBS (i.p.) administration. Biochemical analysis was performed $12 \mathrm{~h}$ after injections. 

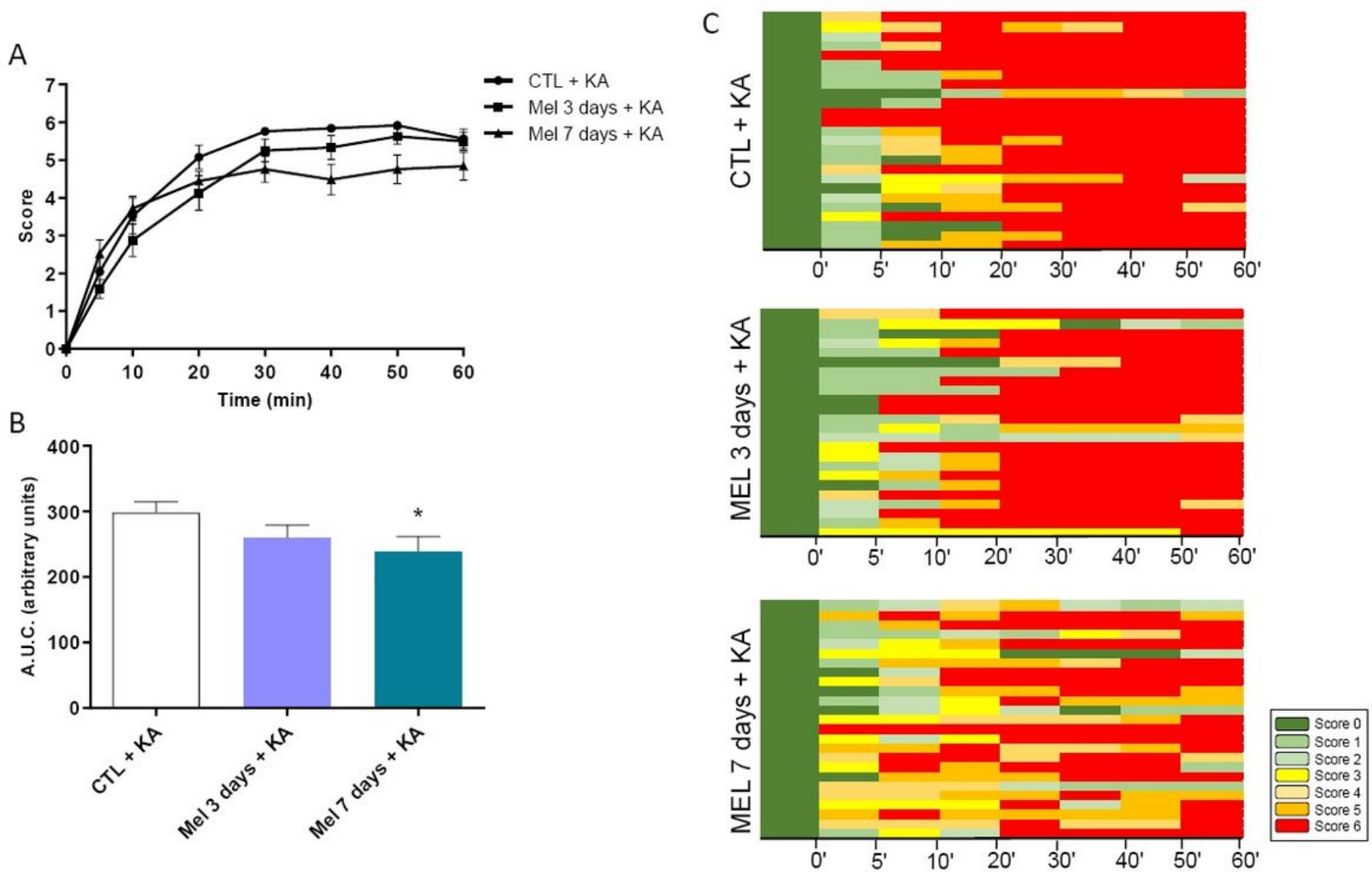

Figure 2

Effects of melatonin pretreatment ( 3 and 7 days) on KA-induced seizures in zebrafish. (A) Seizure-like behavioral scores across time during $60 \mathrm{~min}(\mathrm{~B})$, and intensity evaluated by area under the curve (AUC) for each time of pretreatment. (C) Representative heat maps showing the individual scores across the 60min exposure period of each individual animal. Seizure intensity was represented as mean \pm S.E.M and analyzed by one-way ANOVA followed by Tukey's test. Asterisks above bars express significant differences compared to the CTL $+K A$ group $(n=20-24$ animals per group; $* p<0.05)$. 

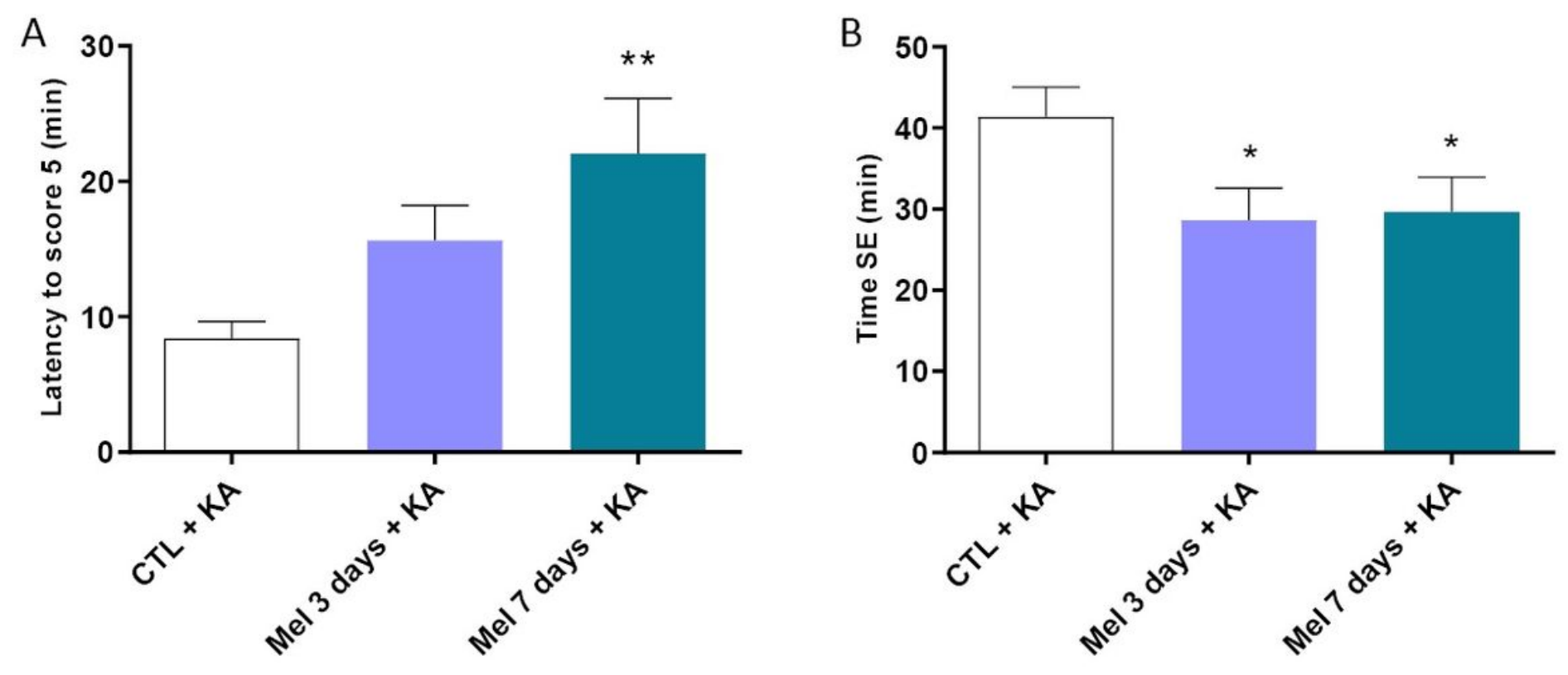

Figure 3

Effects of melatonin pretreatment ( 3 and 7 days) on KA-induced seizures in zebrafish. (A) Latency to score 5 across time during $60 \mathrm{~min}$ (B) Time of SE (duration of 5 and 6 scores) and intensity evaluated by the area under the curve (AUC) for each time of pretreatment. Data were represented as mean \pm S.E.M and analyzed by one-way ANOVA followed by Tukey's test. Asterisks above bars express significant differences compared to the CTL +KA group $\left(n=20-24\right.$ animals per group; $\left.{ }^{*}<<0.05 ;{ }^{* *} p<0.01\right)$. 


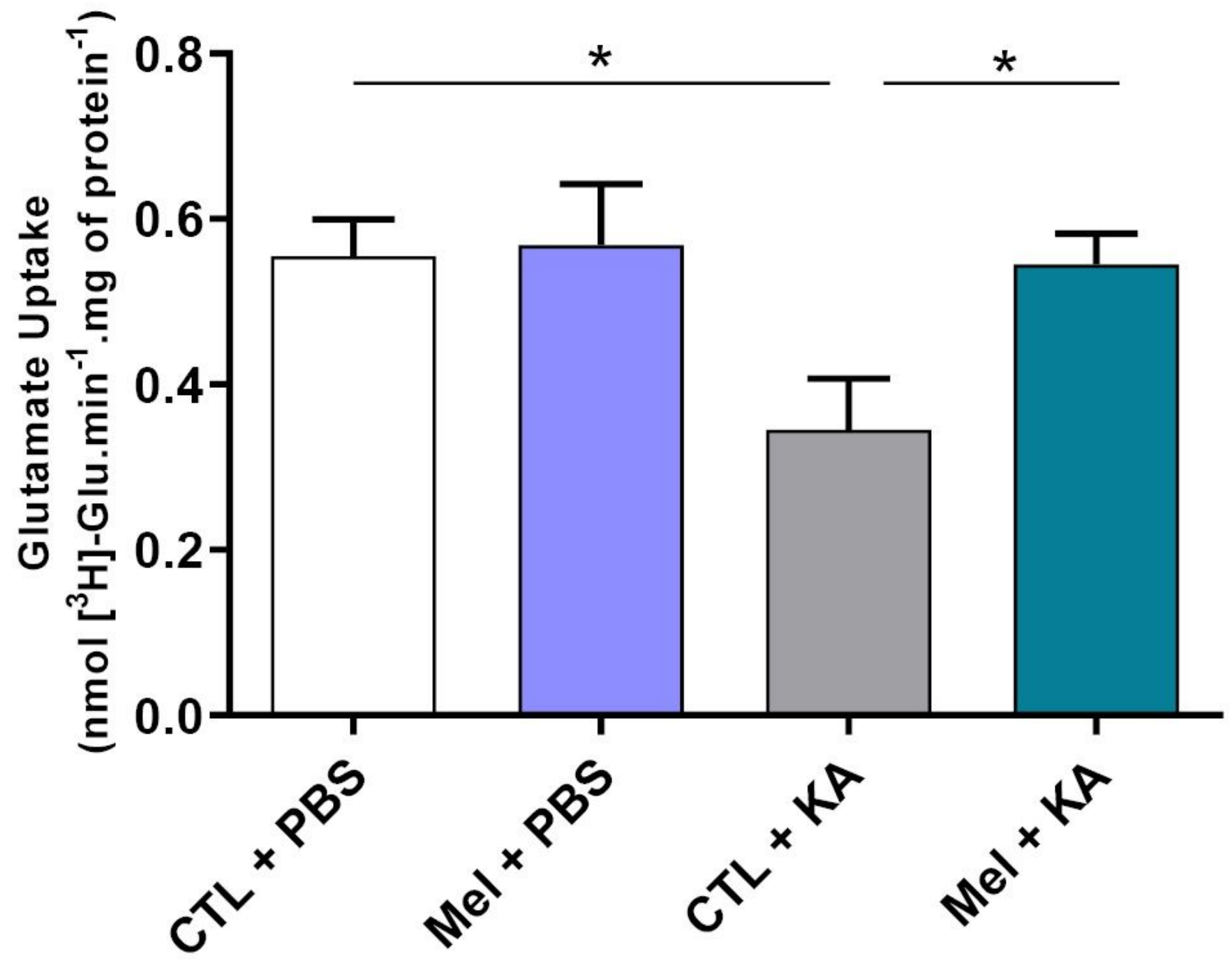

Figure 4

Glutamate uptake in CTL + PBS, Mel + PBS, CTL + KA and Mel + KA groups. The results represent the mean \pm S.E.M $(n=6)$, each in duplicate. The data were analyzed by two-way ANOVA followed by Tukey's post hoc test. ${ }^{*} \mathrm{p}<0.05$. 

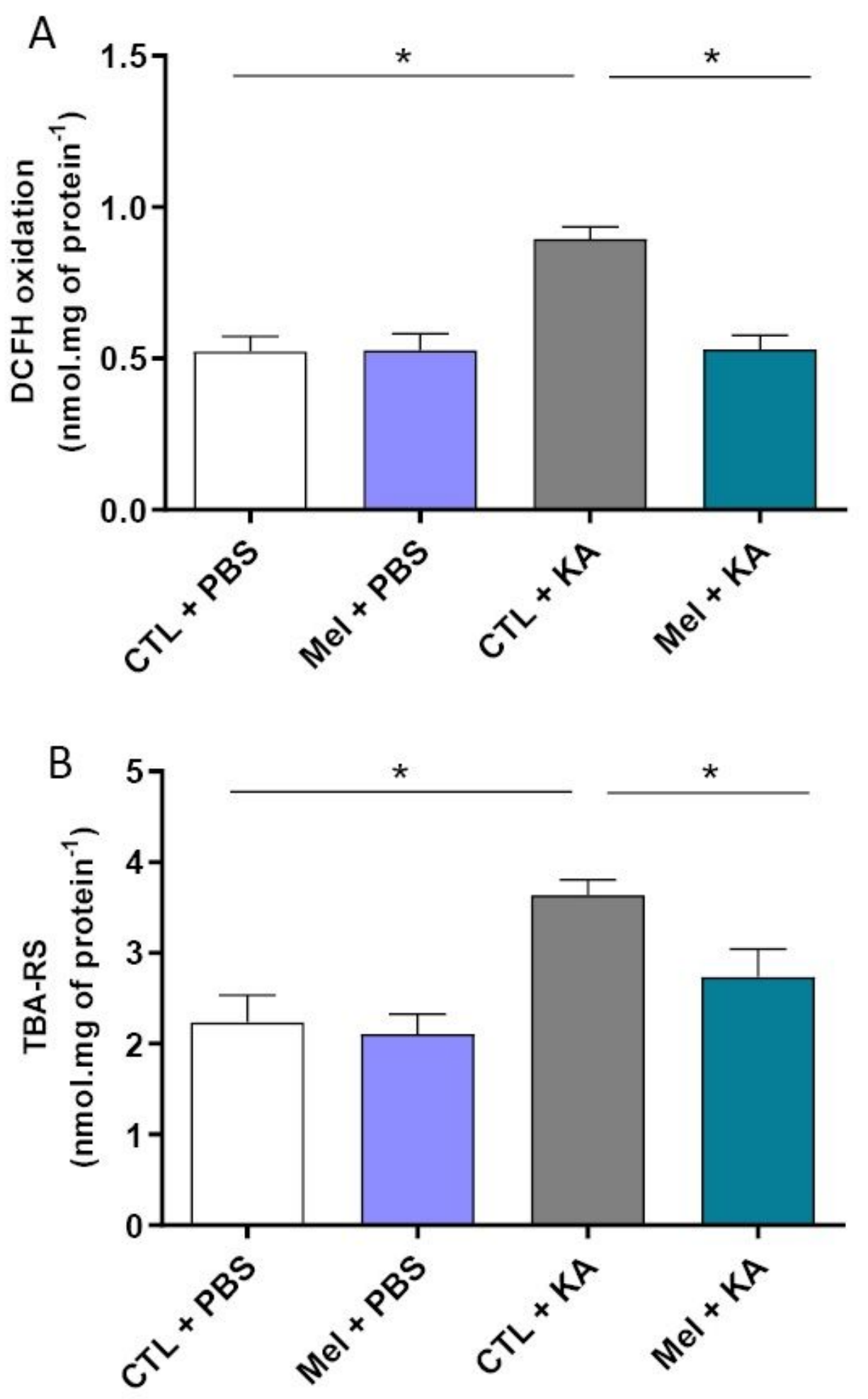

Figure 5

Effect of melatonin treatment before KA-induced seizures on 2',7'-dichlorofluorescein (DCFH) oxidation (A) and thiobarbituric acid levels (B). Results are expressed as mean \pm S.E.M $(n=6)$, each performed in triplicate. The data were analyzed by two-way ANOVA followed by Tukey's post hoc test. ${ }^{*} p<0.05$. 

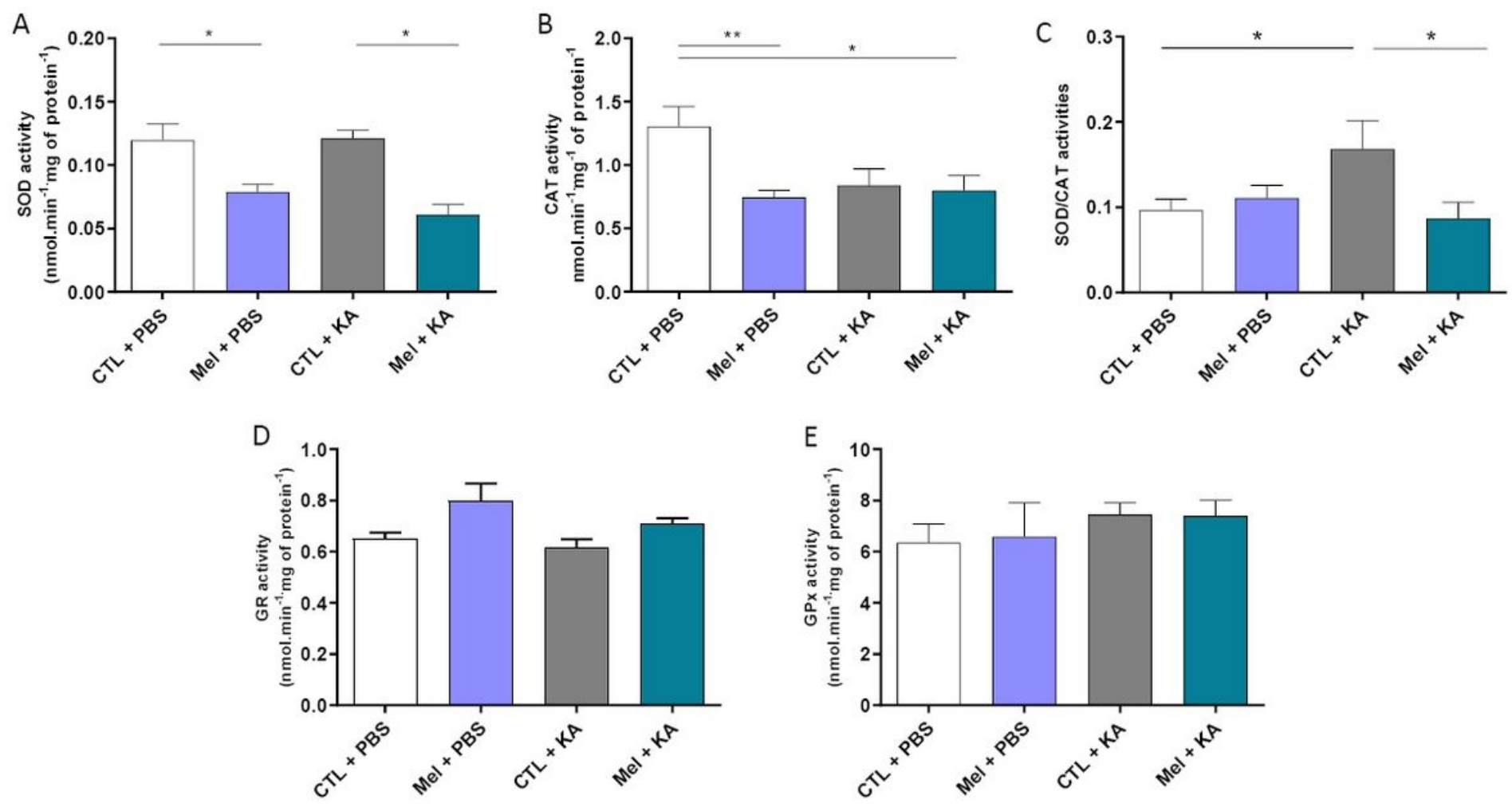

Figure 6

Effect of melatonin pretreatment on KA-induced seizures on the activity of superoxide dismutase (SOD) (A), catalase (CAT) (B), SOD/CAT ratio (C) glutathione reductase (GR) (D) and glutathione peroxidase $(G P x)(E)$. Results are expressed as mean \pm S.E.M $(n=6)$, each performed in triplicate. The data were analyzed by two-way ANOVA followed by Tukey's post hoc test. ${ }^{*} p<0.05$; $* \star p<0.01$.

\section{Supplementary Files}

This is a list of supplementary files associated with this preprint. Click to download.

- MNFariasetalGraphicalAbstract.pdf 